\title{
Sweet's syndrome associated with cellulitis - a challenging diagnosis $^{*}$
}

\author{
Cristina Resende ${ }^{1}$ \\ Teresa Pereira ${ }^{1}$
}

\author{
Rui Santos ${ }^{1}$ \\ Celeste Brito ${ }^{1}$
}

\begin{abstract}
Sweet's syndrome is a neutrophilic dermatosis with worldwide distribution that has been associated with inflammatory autoimmune diseases, infections, malignancies, drugs, and pregnancy. The disease is idiopathic in up to $50 \%$ of patients. A 64-year-old woman, diagnosed with right limb cellulitis (4 days of evolution), was seen at our department, due to persistent cellulitis and progressive appearance of painful nodules and plaques in both shins and the right forearm (2 days of evolution). Taken together, clinical, laboratory and pathological data suggested the diagnosis of Sweet's syndrome, probably secondary to cellulitis of the right inferior limb. We suggest that cellulitis may be associated with Sweet's syndrome, a rare association in the literature.
\end{abstract}

Keywords: Cellulitis; Infection; Sweet syndrome

\section{INTRODUCTION}

Sweet's syndrome (SS) has worldwide distribution, without racial predilection but with a female predominance of 4 to 1 and an average onset age of $30-60$ years. ${ }^{1,2,3}$ It has been associated with inflammatory autoimmune diseases, infections, malignancies, especially acute myelogenous leukemia, drugs, and pregnancy., ${ }^{4,5}$ The disease is idiopathic in up to $50 \%$ of patients. ${ }^{1,6}$

Initial cutaneous lesions are tender, nonpruritic, erythematous papules and plaques, with a pseudovesicular appearance. ${ }^{1,2}$ Patients may present with constitutional signs and symptoms, such as fever and malaise ${ }^{1}$. Lesions predominantly favor the head, neck and upper extremities, though they can occur anywhere. ${ }^{1,5}$ Typical histological features include a dense, perivascular, neutrophilic infiltrate, edema and leukocytoclasia without evidence of vasculitis. ${ }^{1,5}$ Leukocytosis and increased erythrocyte sedimentation arises in the majority of SS patients. ${ }^{5,6}$

\section{CASE REPORT}

A 64-year-old woman, diagnosed with cellulitis of the right inferior limb (4 days of evolution) was seen at our department due to persistent cellulitis and progressive appearance of painful nodules and plaques in both shins (2 days of evolution). She had previously been treated with cefatrizine $500 \mathrm{mg} 4$ times daily, without any improvement. The patient reported that the lesions appeared about 4 days later, following a trauma caused by a metal object. Physical examination revealed erythema, edema, heat and pain in the right inferior limb. The redness had a welldemarcated but irregular border and spared portions of skin in an unpredictable pattern. The inflamed area was edematous, creating a plaque that pitted on pressure. Moreover, the patient had multiple, infiltrated, painful, erythematous, violaceous nodules and plaques of variable sizes, measuring $1-7 \mathrm{~cm}$ in diameter, with a vesicular or bullous appearance, localized in both shins (Figure 1). In addition, she had painful, reddened eyes. 


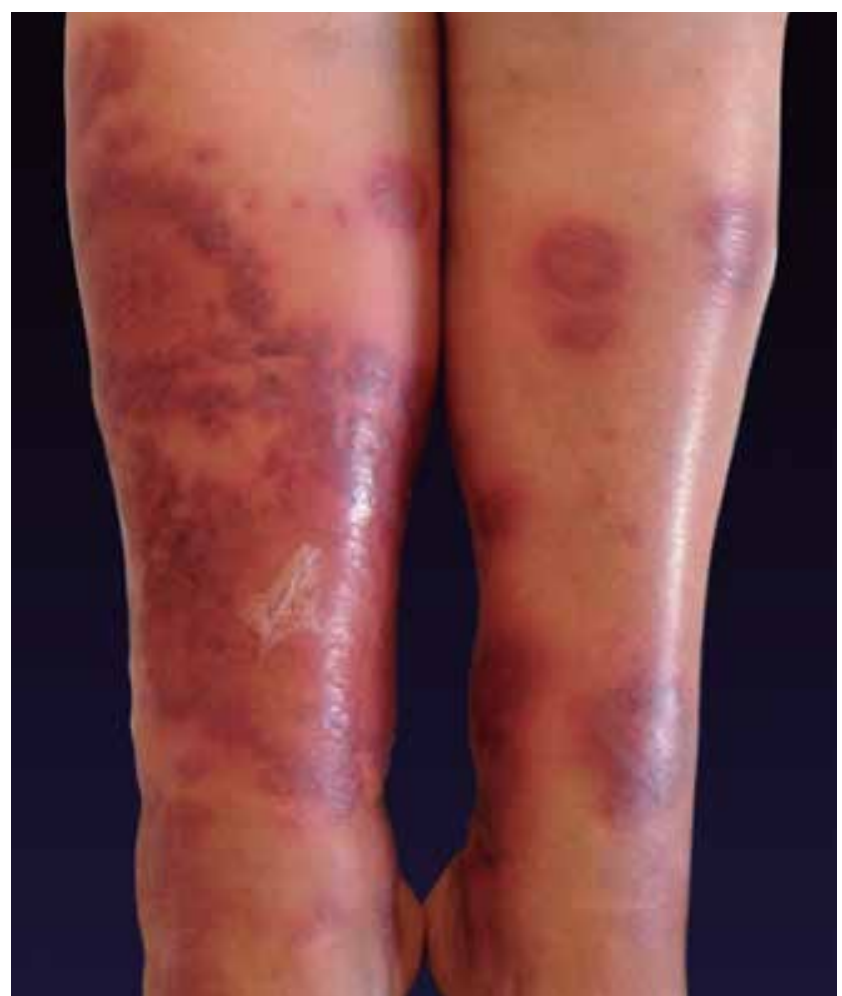

FIGURE 1: Erythema, edema of the right inferior limb and multiple, infiltrated, painful erythematous, violaceous nodules and plaques, of variable sizes, measuring $1-7 \mathrm{~cm}$ in diameter, with a vesicular or bullous appearance, localized in both shins.

Her medical history was unremarkable and there was no family history of cutaneous diseases. She was not taking any medication prior to the diagnosis of cellulitis in the right inferior limb. She denied fever and she was afebrile at our observation.

Laboratory examination revealed leukocytosis (white blood cells $11700 / \mathrm{uL}$, with a high absolute neutrophils count $(78 \%)$ and an increased C-reactive protein level of $159 \mathrm{mg} / \mathrm{L}$.

Histopathologic examination of an incisional biopsy on a lesion taken from the left leg revealed edema in the papillary dermis with dense inflammatory infiltrate in the upper dermis, consisting mainly of mature neutrophils without fibrinoid necrosis (Figure 2).

The patient underwent extensive investigation, including a CT of the chest, abdomen and pelvis, esophagogastroscopy, colonoscopy and mammography. This extensive workup revealed no focus of infection or malignancy.

Taken together, clinical, laboratory and pathological data suggested the diagnosis of SS, probably secondary to cellulitis of the right inferior limb.

The medication was changed from cefatrizine to penicillin G 20 million units, divided into 4 daily doses over 14 days. Moreover, she was given

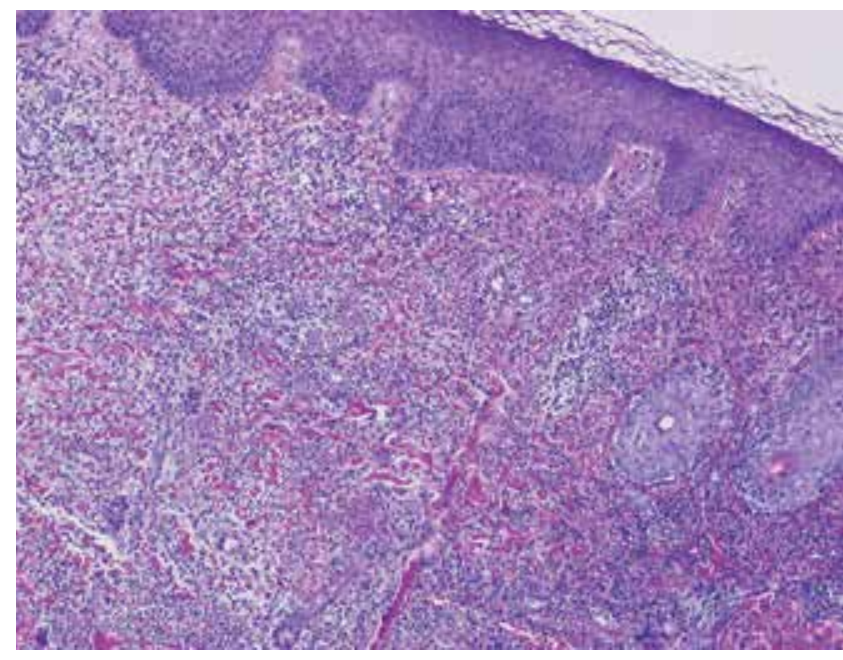

FIGURE 2: Edema of the papillary dermis with dense inflammatory infiltrate in the upper dermis, consisting mainly of mature neutrophils without fibrinoid necrosis (haematoxylin and eosin, magnification 400x).

prednisone $0.5 \mathrm{mg} / \mathrm{kg} /$ daily, tapered by $5 \mathrm{mg}$ every 4 days, resulting in significant improvement of the skin lesions within 2 weeks, without residual scarring.

During the 18 months of follow-up, the patient did not experience recurrence of skin lesions.

\section{DISCUSSION}

The etiology of SS is still unknown but the association with inflammatory autoimmune diseases, infections, malignancies, drugs, and pregnancy suggests a hypersensitivity reaction. 1,7,8 Another hypothesis is that a local or systemic dysregulation of cytokine secretion, including interleukin-1, interferon gama, granulocyte colony-stimulating factor (G-CSF) or granulocyte-macrophage colony-stimulating factor (GM-CSF), contributes to the pathogenesis of SS. ${ }^{1,7}$

SS has been linked with a wide range of infections, including upper respiratory tract infections, due to Streptococcus, the most common infection associated with SS and gastrointestinal yersiniosis described in the literature. ${ }^{1,5,6}$

In our case, we suggested that SS was probably secondary to cellulitis of the right inferior limb.

Furthermore, SS has reportedly been caused by a wide variety of drugs, including granulocyte colony-stimulating factor, antibacterials, retinoids, antiepileptics, antihypertensives, oral contraceptives and vaccines. ${ }^{1,5}$ Drug-induced SS is rare and affects predominantly middle-aged women, with skin lesions usually appearing 5-7 days after the first administration of the drug. ${ }^{1,3,4}$ The most common localization of druginduced SS was the arms, followed by the legs, face, neck and trunk. ${ }^{1,3,4,7}$ 
Our patient was not on any medication before the diagnosis of cellulitis in the right inferior limb but she was medicated with cefatrizine $500 \mathrm{mg}$, two days prior to the onset of SS lesions. Consequently, another, less likely, hypothesis, is that our patient had drug-induced SS caused by cefatrizine. It is less probable because the temporal relationship between drug ingestion and the clinical presentation of SS was only two days after the onset of antibiotherapy. Furthermore, the patient saw significant improvement in skin lesions within 2 weeks, after our first observation. In addition to discontinuing cefatrizine, penicillin $G$ and prednisone were administered to the patient, which could explain the rapid improvement in the lesions.

Our patient also underwent an extensive investigation to exclude other causes of SS, which revealed no specific findings.

The presence of neutrophilia, common in most SS patients, including our patient, was observed in a few patients with drug-induced SS, described in the literature. ${ }^{3,4,5,7}$ Ocular involvement, also observed in our patient, is more frequent in classic SS than in druginduced SS.,

To our knowledge, Dinh $\mathrm{H}$. et al. reported the first-ever published case of SS that was likely secondary to cellulitis, involving a 38-year-old woman, who developed SS following cellulitis in her left leg. ${ }^{1}$ Our patient also developed cellulitis in the lower limb; two days later, she developed SS in the shins and forearms. We identified precisely the simultaneous presence of both diseases. The cellulitis in her right limb improved quickly within a few days, while the SS improved following the institution of antibioterapy with penicillin and systemic corticotherapy.

We suggest that cellulitis, a frequent cutaneous infection, may be associated with SS, an unusual association in the literature. However, we cannot exclude the hypothesis that SS is a mimicker of cellulitis called "giant cellulitis-like SS", recently described in the literature as a possible new variant of neutrophilic dermatosis. ${ }^{2} \square$

\section{REFERENCES}

1. Dinh H, Murugasu A, Gin D. Sweet's syndrome associated with cellulitis. Australas J Dermatol. 2007:48:105-9.

2. Surovy AM, Pelivani N, Hegyi I, Buettiker U, Beltraminelli H, Borradori L. Giant cellulitis-like Sweet Syndrome, a new variant of neutrophilic dermatosis. JAMA Dermatol. 2013;149:79-83.

3. Clark BM, Homeyer DC, Glass KR, D'Avignon LC. Clindamycin-induced Sweet's syndrome. Pharmacotherapy. 2007;27:1343-6.

4. Kalai C, Brand R, Yu L. Minocycline-induced Sweet syndrome (acute febrile neutrophilicdermatosis). J Am Acad Dermatol. 2012;67:e289-91.

5. Wojcik AS, Nishimori FS, Santamaría JR. Sweet's syndrome: a study of 23cases. An Bras Dermatol. 2011;86:265-71.

6. Abramovits W, Stevenson LC. Sweet's syndrome associated with Staphylococcus aureus. Int J Dermatol. 2004;43:938-41.

7. Sáez M, García-Bustínduy M, Noda A, Dorta S, Escoda M, Fagundo E, et al. Druginduced Sweet's syndrome. J Eur Acad Dermatol Venereol. 2004:18:233.

8. Rochet NM, Chavan RN, Cappel MA, Wada DA, Gibson LE. Sweet syndrome: clinical presentation, associations, and response to treatment in 77 patients. J Am Acad Dermatol. 2013;69:557-64.

\author{
MAILING ADDRESS: \\ Cristina Resende \\ Department of Dermatology and Venereology \\ Hospital de Braga \\ Sete Fontes - São Victor. \\ PT-4710-243 Braga, Portugal. \\ E-mail: cristinapresende@gmail.com
}

How to cite this article: How to cite this article: Resende C, Santos R, Pereira T, Brito C. Sweet's syndrome associated with cellulitis - a challenging diagnosis. An Bras Dermatol. 2016;91(1):94-6. 